\title{
Editorial
}

\section{MicroRNA-A Critical Regulator in the Pathogenesis and Malignant Transformation of Oral Submucous Fibrosis}

Molecular analysis has provided us vital data, unveiling the complex pathways governing the pathogenesis of oral submucous fibrosis (OSMF). Both the pathogenesis (collagen accumulation) and malignant transformation of OSMF are attributed to arecoline, a major component of areca nut. The accumulation of collagen follows two major pathways: increased production of collagen due to over expression of cytokines like TGF- $\beta$, decreased degradation of collagen due to increased secretion of tissue inhibitors of matrix metalloproteinases (TIMPs) and reduced secretion of matrix metalloproteinases (MMPs). The role of areca nut has been extensively studied with animal models and is proven to be a carcinogen. ${ }^{1,2}$ Several studies correlating the role of microRNA (miRNA) in the initiation and progression of fibrosis in visceral organs $\mathrm{s}^{3,4}$ has raised the possibility of miRNA's role in the pathogenesis of OSMF. MicroRNA modulates the expression of multiple genes by post-transitional regulation. It plays a major role in fibrosis by controlling the differentiation of fibrotic cells and/or regulating the translation of extracellular matrix components. ${ }^{5,6}$ The epithelial-mesenchymal transition (EMT) is responsible for multiple biological process, including organ fibrosis, cancer initiation and progression. MicroRNAs regulate both the transcriptional and post-transcriptional regulatory mechanisms of EMT, thus, may serve as biomarkers and therapeutic targets for EMT-based pathological conditions, including OSMF. Transforming growth factor (TGF) is considered to be a major mediator of fibrosis. It enhances the expression of connective tissue growth factor (CTGF), which in turn enhances the signalling of TGF and multiple profibrotic factors, like vascular endothelial growth factor (VEGF), insulin-like growth factor-1 (IGF-1), integrins, Wnts and endothelin-1 (ET). ${ }^{5}$ Studies have shown that at least 10 miRNAs play a major role in signal regulation of TGF- $\beta$ /CTGF. Upregulation of profibrotic miRNA or downregulation of antifibrotic miRNA may lead to fibrosis. ${ }^{5-7}$ Thus, identifying the miRNA signature of OSMF patients will be of diagnostic and therapeutic significance. Binjie LIU et al demonstrated changes in miRNA signature in normal mucosa before and after treatment with arecoline. The study also showed that the miRNA expression was reversed after treating the OSMF tissue with saliva combined with low doses of prednisolone. ${ }^{8}$ Cervigne et al compared the miRNA signature of leukoplakia (progressive and nonprogressive) with oral squamous cell carcinoma (OSCC) and found that progressive leukoplakia and OSCC showed significant difference when compared with nonprogressive leukoplakia and normal oral mucosa. ${ }^{9}$ Dang et al compared the miRNA-137 promoter methylation in oral lichen planus (OLP) and OSCC. The result showed significant difference in the miRNA pattern between normal mucosa and patients with OLP/OSCC. ${ }^{10,11}$ The study also showed a lack of significant difference in the miRNA pattern between patients with OLP and OSCC patients, substantiating OLP's inclusion as an oral potentially malignant disorder (OPMD). Thus, analysing the miRNA signature of OSMF may also aid in accessing its malignant potential. Further, creating multiple miRNA profiles using large scale samples will provide us with a template for monitoring the disease progression and treatment response.

\section{REFERENCES}

1. Ekanayak RP, Tilakaratne WM. Oral submucous fibrosis: review on mechanisms of pathogenesis and malignant transformation. J Carcinogene Mutagene 2013;S5:002. DOI:10.4172/2157-2518.S5-002.

2. Rajendran R, Sukumaran A. Editorial. Oral submucous fibrosis: revised hypotheses as to its cause. J Contemp Dent Pract 2013 Sep 1;14(5):i-iii.

3. Babalola O, Mamalis A, Lev-Tov H, Jagdeo J. The role of microRNAs in skin fibrosis. Arch Dermatol Res 2013 Nov;305(9): 763-776.

4. Srivastava SP, Koya D, Kanasaki K. MicroRNAs in kidney fibrosis and diabetic nephropathy: roles on EMT and EndMT. Hindawi Publishing Corporation BioMed Research International. 2013;Article ID 125469:10.

5. Vettori S, Gay S, Distler O. Role of MicroRNAs in fibrosis. The Open Rheumatology Journal 2012;6:130-139.

6. Jiang X, Tsitsiou E, Herrick SE, Lindsay MA. MicroRNAs and the regulation of fibrosis. FEBS J 2010 May;277(9):2015-2021.

7. Zhao X, Lu Y, Nie Y, Fan D. MicroRNAs as critical regulators involved in regulating epithelial- mesenchymal transition. Current Cancer Drug Targets 2013;13:935-944.

8. Binjie LIU, Jun C, Xinchun J. Changes of miRNA after oral submucous fibrosis cocultured with salvia and low-dose prednisolone. J Cent South Univ (Med Sci) 2014;39(5):471-476. 
9. Cervigne NK, et al. Identification of a microRNA signature associated with progression of leukoplakia to oral carcinoma. Hum Mol Genet 2009 Dec 15;18(24):4818-4829.

10. Dang, et al. MicroRNA-137 promoter methylation in oral lichen planus and oral squamous cell carcinoma. J Oral Pathol Med 2013;42: 315-321.

11. Patil S, Rao RS, Raj AT. Editorial. Role of miRNA in the malignant transformation of oral lichen planus. J Contemp Dent Pract 2015; 16(2):i-ii.

Shankargouda Patil

Associate Professor

Department of Oral Pathology and Microbiology

Faculty of Dental Sciences

MS Ramaiah University of Applied Sciences

Bengaluru, Karnataka, India

Roopa Rao

Professor and Head

Department of Oral Pathology and Microbiology

Faculty of Dental Sciences

MS Ramaiah University of Applied Sciences

Bengaluru, Karnataka, India

Thirumal Raj

Final Year Postgraduate Student

Department of Oral Pathology and Microbiology

Faculty of Dental Sciences

MS Ramaiah University of Applied Sciences

Bengaluru, Karnataka, India 